\title{
Editorial
}

Annals of
Neurosciences

Published online: September 9, 2016

DOI: $10.1159 / 000449176$

\section{International Yoga Day 2016: A Call for Analysis of One Month Training Program on the Health Benefits of PM's Call for Yoga}

\author{
Akshay Anand Natasha Sayal \\ Neuroscience Research Laboratory, Department of Neurology, Post Graduate Institute of Medical Education and \\ Research (PGIMER), Chandigarh, India
}

Mind-body therapies, which include Yoga, Tai chi, Qigong etc. are reemerging as beneficial tools for providing solutions to the postmodern healthcare challenges. These therapies are believed to address the mind-body interaction by either inducing parasympathetic systems or molecular mechanisms hitherto undeciphered. Among these mind-body therapies, yoga is considered as a fast growing and popular system of asanas and breathing practices which allow seamless integration of physical exercises with meditative postures. On the eve of International Yoga Day on June 21, Indian Prime Minister Sh Narendra Modi decided to perform yoga along with 35,000 citizens of Chandigarh at its Capitol complex. This has led the local administration into a series of activities, which included Yoga for good life conclave and a call to analyse the changes in health parameters resulting from AYUSH common Yoga protocol, as thousands thronged to join the PM in performing yoga on June 21, 2016.

Emerging studies have shown the positive effect of yoga and exercise on the physiological, biochemical, cognitive, and molecular changes, although additional controlled studies need to be carried out. The precise molecular mechanism underlying yoga, therefore, remains unclear. The proposed study in the beautiful city (or city of parks) is in support of the hypothesis that these mind-

\section{KARGER}

E-Mail karger@karger.com

www.karger.com/aon body interventions must have measurable regenerative and molecular effects that need the same rigour of scientific investigation and analysis reserved for orthodox pharmacological discovery. The non-pharmacological battery of yogic exercises is proposed by the PM to be standardised through Quality Council of India, facilitating knowledge generation regarding homeostasis of the mind and body. However, a study commissioned at such a short notice is going to be a herculean task for the scientists and administrators of Chandigarh. Any dysregulation of brain and body are believed to result in a cumulative effect that might result in various diseases in later life whose signatures are hypothesised to appear in the 1-month training program even though pharmacologist continue to oppose the wellness regimen. As mentioned in the Taittiriya Upanishad (Gambhirananda, 1986), Indian vedic textual source, the human frame has 5 bodies which are physical, vital, mental, intellectual, and blissful sheath. These are affected by wrong postural habits, sedentary lifestyle, wrong breathing pattern, mental afflictions and occupational mental stressors, work load, and psychosomatic ailments, respectively. All of these are believed to occur through underlying cellular and molecular dynamics responsible for bringing perceived clinical or neuropsychological benefits. Yoga is effective in
(C) 2016 S. Karger AG, Basel

0972-7531/16/0233-0129\$39.50/0
Akshay Anand

Neuroscience Research Laboratory, Department of Neurology

Post Graduate Institute of Medical Education and Research (PGIMER) Chandigarh 160012 (India)

E-Mail akshaylanand@ rediffmail.com 
coping with stress; it reduces anxiety and brings changes in the levels of neurotransmitters; it helps in the regulation of inflammatory molecules, thereby reducing the heart rate, maintaining systolic and diastolic blood pressure. The Prime Minister characterizes yoga to 'zero budget assurance' for good health. Certain changes in the salivary proteasome have also been documented through isolated studies. Despite these and various other studies, the exact mechanism underlying these changes remains unclear and unverified. Studies related to endurance exercises have provided indirect evidence documenting an increase of neurotransmitters, neurogenesis, angiogenesis, neurotropic factors. Increase in cognitive functions, delay in ageing, altered gene expression, cell proliferation, cytokine secretion have not been comprehensively analysed or correlated due to challenges of the prospective study. As majority of these molecules participate in cell survival mechanisms, it remains to be seen whether yoga can modulate regeneration and strengthen the repair system through cellular and molecular processes in larger cohorts. Regardless of the proposed hypothesis, the Indian PM insists that yoga does not call for a personal gain but seeks freedom from material life and instills a spirit of working for others. Nevertheless, with the launch of world's biggest yoga clinical trial by Patanjali Research Foundation, together with the advent of modern molecular techniques such as microarray analysis, real-time PCR analysis and whole genome sequencing, there is a grow- ing curiosity to undertake studies which allow correlation of the genomic, proteomic, and epigenomic changes in the blood of yoga practitioners. If such molecular studies are conducted on yoga practitioners, at various time points with long-term clinical outcomes, it may reveal information relevant to cause and effect of yoga. Such studies need larger cohorts and compliance of healthy volunteers. In some retrospective studies, Qigong practice and Sudarshan Kriya have been documented to cause molecular changes in blood cells by employing PCR and microarray analysis, in an effort to map the molecular changes, if any. However, the scientific rigour has always been lacking in majority of such studies. Ironically, these studies have either been undertaken by those who were formally not trained in molecular biology or those who had a cognitive bias against the utility of yoga as a tool for preventive or curative healthcare. The latter insist that yoga studies should be limited to diseased subjects, ignoring the utility of T1/T2 translational loop. Interestingly, a study focused on endurance exercises in healthy volunteers has shown an increase in the pool of hematopoietic stem cells and endothelial progenitor cells in bone marrow and peripheral blood. However, stem cell mobilization-related markers or proteomic analysis resulting from yogic breathing techniques, have been conspicuous by their absence. The Indian neuroscientists look forward to mapping the molecular, neuropsychological, and pathophysiological basis of yoga and its effects. 